\title{
Study on the Training of Core Strength in the Competitive Aerobics Sports
}

\author{
Chao Fan \\ Xi’an Physical Education University, Xi'an, Shaanxi , 710068, China
}

Keywords: Competitive aerobics; Core strength; Training method

\begin{abstract}
Core strength of the aerobics refers to a kind of ability that can stabilize the core parts of the body, control the center of gravity and transfer the upper and lower extremity strength through targeted training. It can provide the aerobics athletes with stability and supports when they complete sets of action. Therefore, discussion on strength training of aerobics athletes plays an important role in improving their technical level. Based on the study and practical experience of the author, this paper firstly analyzed the training value that core strength training applied in competitive aerobics, and then put forward the training methods of competitive aerobics core strength.
\end{abstract}

\section{Introduction}

Competitive aerobics is a sport which performs a set of continuous, complex and high-strength action accompanied by music. The entire action shows the flexibility, strength, the diversity of the seven basic steps and the ability of completing sets of action sports combined with difficult movement through continuous combined movement. The competitive aerobics competition rules (2009-2012) clearly put forward that the requirements on movement and difficulty of diversity, complexity and innovation, which more prominently highlights project characteristics and personality characteristics of aerobics athletes. Only the continuously uses and improves the overall quality and fitness of athletes, strengthens the core strength training of athletes, can athletes ensure perfect completion of actions. Core strength is a kind of force due to shrinkage with muscles and ligaments attaching at the core parts of the human body under the control of the nervous system. The core muscles usually refers to what we call the trunk, which is close to the center of gravity of the body intermediate links, including the spine, pelvis and surrounding muscles. In this paper, we used the theory of knowledge combined with the long-term practice of competitive aerobics training to put forward the views on core strength training and improving the competitive aerobics effect in order to provide reference for the scientific athletic aerobics training.

\section{Training Value of Core Strength Training in Competitive Aerobics}

Core strength training is the foundation to improve the competitive ability of athletes. Only fully embodying and performing in the competitive aerobics special technologies, can a good core strength level has the real meaning. Core strength training can help athletes better grasp of advanced and reasonable sports technology. For example, the right angle support. If there is no strong core strength, the arm strength is impossible to complete the difficulty. Actually, the purpose of the core strength training is to promote the organization coordination and all-round development of athletes organism, organ and system function so that to adapt to the different requirements of different special athletes organism function. However, these special requirements on special athletic ability, sport technical and tactical training are impossible. In order to promote the athletes to master more complex advanced motion technology, we must combine with special training in core strength training synchronously. Therefore, the core strength training is the basis of technical training, tactical training and improving athletic performances. 
Core strength training is the guarantee to improve the performance of athletes. The core strength is the ubiquitous role in competitive aerobics. Whether the aerobics basic movement technology or difficult movement technology, they are closely linked with the core strength. Core strength is the important "source" and "power-borrow source" in the movement when people make difficult action without any supports. In this process, the core muscles function as the stability of the center of gravity, forcing in linkage and force transmission. At the same time, it is also the main part of the overall force, and plays a pivotal role in connecting the upper and lower limb on coordination and integration effort. Therefore, the core strength is the guarantee for competitive aerobics athletes to get excellent grades.

Core strength training is the process to cultivate athletes' good psychological quality and tenacious will. The intense competition of competitive aerobics requires a high expectation for athletes' psychological quality. The core strength training is helpful to cultivate the psychological quality to adapt to competition. At the same time, the indomitable will of the training is based on the physical development of the athletes. Core strength training is the extremely difficult process to improve and enhance the function of human motion, and gradually achieve the specific performance objectives and requirements. This process will profoundly affect the quality of people. To enhance the depth of human muscle and small muscle strength, the core strength training needs to control the body for a long time under unbalanced conditions. We also need to overcome the physical and psychological barriers. The athletes must have the determination and perseverance, and do not afraid of bitter and insist for a long time, which can be effective in the end. Therefore, it is helpful to cultivate athletes hard unremittingly ideological style, helps cultivate athletes good psychological quality and establishes athlete's indomitable willpower.

Core Strength Training Has a Positive Effect on the Prevention of Injuries and Prolong Sports Life of Athletes. To complete the C type difficult movement, athlete's the body completes the rotation and other action without support in the air. The core strength is not only an important basis to ensure the completion of the air movement and posture of athletes to maintain, but also the the indispensable power guarantee to avoid the damage in landing. Aerobics athletes are landed by two kinds of loads including the compression and distortion. The compression load is equivalent to 10 times their own weight. If the gravity is slightly not stabilize, it will cause failure. This not only lost the gold medal but also lead damage caused by movement due to the incorrect landing. We can strengthen the erector spinal muscle strength through the core strength training and enhance the ability to rotate around the rotating shaft of the body so that to keep the balance and stability of the body when falling in the ground. At the same time, the training injury can be greatly reduced. This will ensure the duration of the training system of high level athletes and sports ability, thus can prolong the sports life of athletes effectively.

\section{The Training Methods of Competitive Aerobics Core Strength}

A single practice without the aid of any instrument. This kind of practice is the basic means of competitive aerobics core strength training, which can make athletes deeply understand the core parts of the force and effective control of the body. For example, pushing up control, supine mastering and lateral position. It is very helpful to complete the difficult movement, such as pushing ups, felling to the ground, leg spinning and cutting. When exercise the arm supporting and legs stretching, we should keep the trunk straight, and take breath without holding your breath. The scale of the training load is decided on supporting time; while the standard of action will affect the quality of training.

To practice by using unstable equipment. Using unstable equipment for core strength training is a commonly used method of competitive aerobics training. Unstable instruments include balanced ball, Swiss ball, balance board and suspension rope. The use of this type of equipment for strength training can effectively mobilize the participation of deep muscle movement, and control the body in the course of action to keep correct posture. In actual exercise, it simultaneously make 
players keep closer to the body balance in force. There are a lot of practice methods using unstable instruments. Let's take the Swiss Ball as an example. Pushing-up controlling with legs or single leg on the Swiss Ball to constantly stimulate core muscles to maintain the correct posture through the instability of the Swiss Ball so that to achieve the purpose of enhancing the core muscles control force. Supine-clipping Swiss Ball exercise and waist strength training by straight leg or leg lifting can train both of lumbar muscles and the core muscles. The training has the similar requirement on the aerobics exercises, such as tuck, pike, kesake and straitening, etc. Athletes must be clearly visible in the air posture, and trunk must at the standard position. Similarly, the pelvis must in a fixed position. If you sit on a Swiss Ball, you must twist your hip and divide the leg paralleling with the ground; moreover, one hand is in front of your body, while the other one is behind your body and the spine must be strong and straight. This training can be used as the basis of the training of competitive aerobics. This is because many actions such as basic steps, dynamic strength and static force action are required to hold this position for upper body of athletes. If you want to increase the difficulty, you can place the balance disc under the Swiss Ball. In fact, all of the special strength training in the land (flat) can be carried out in unstable equipment. The evaluation of load is time. Some load of actions can be accounted by double index, including time and count. For example, if an athlete lies supine at pad with a leg on a Swiss Ball and his arms are straight supporting the ground in 90 degrees or the other foot ankle tied lead belt lifting the weight leg to make rotation of the hip movements and abduction movements, his number and time of support can be used as load evaluation index.

Weight-bearing exercises under the unbalanced state. The characteristics of core strength training of athletes is a kind of strength training under an unbalanced and unstable state. Under various load conditions, the purpose is to stimulate and improve the balance and control ability of the neuromuscular system through the adjustment of the unstable physical state by itself. Simple weight training can increase training load in the abdominal or lumbosacral (Yang bridge, Side Bridge and Overlooking Bridge) placed in the specific training. The athletes stood in unstable instruments or sit on a Swiss Ball, so that the body is in a state of imbalance. Further, there will be the small load strength exercises, such as standing on a balance disc and both hands should hold the barbell of twisting motion, which requests the athlete to keep the body in the correct position to complete the process of strength training. This training not only exercise the waist muscle, but also make the core muscles maintain a center vertical axis in regulating the body. The training are very close to competitive aerobics in the jump, balance and flexibility of the difficulty. Thus, the training principle is the small load with several times. Static training is generally 30 to 50 seconds; dynamic exercises of each repeated are generally not more than 15 times.

\section{Summary}

The core strength plays an important role in competitive aerobics. Core strength training is an important content of competitive aerobics in athletes physical training; it is also one of the preconditions to improve the physical fitness of athletes and perfect action. To stand in the world strong teams in the ranks of the competitive aerobics, we must strengthen the core strength training. We can show all the beauty of the competitive aerobics accompanied by beautiful music through graceful movements, high-level techniques and ingenious transition and connections. In core strength training, the coaches should adjust the difficulty and load according to the actual situation of athletes, and combine with the characteristics of the project selection training methods of competitive aerobics. Only in this way, can we achieve some actual achievements.

\section{References}

[1] Hu Xiaofang, An Manna. The Exploration on Athletes Core Strength Training of Competitive Aerobics[J]. Journal of Ankang University, 2013, (03): 59-62.

[2] Xiang Yangchun, Yang Jia Peng. The Exploration on Athletes Core Strength and Training of 
Competitive Aerobics[J]. Study and Education on Sports, 2011 (04): 98-102+118.

[3] Yao Jie. Competitive Aerobics athletes core strength training[J]. Hubei Sports Science and Technology, 2011, (03): 334-335.

[4] Zheng Huayan, Zhang Pinghua. Application Research on The Core Strength Training in Competitive Aerobics Training[J]. Hubei Sports Science and Technology, 2010, (06): 698-699.

[5] Wu Fanglei. Analysis on Application of Core Strength Training in Sports[A]. China Association of Biomechanics in Sports Club. The Fourteenth National Sports Biomechanics Symposium Proceedings[C]. Chinese Association of Biomechanics in Sports Clubs:, 2010:3.

[6] Wang Huadi. Analysis on Strength training of Competitive Aerobics[J]. Heilongjiang Science and Technology Information, 2009, (03): 182. 